\title{
Endothelial cell Bcl-2 and lymph node metastasis in patients with oral squamous cell carcinoma
}

\author{
Sandra B. C. Tarquinio ${ }^{1,2}$, Zhaocheng Zhang ${ }^{2}$, Kathleen G. Neiva ${ }^{2}$, Peter J. Polverini ${ }^{3}$, Jacques E. Nör ${ }^{2,4,5}$ \\ ${ }^{1}$ Department of Oral Pathology, Federal University of Pelotas, Pelotas, RS, Brazil; ${ }^{2}$ Angiogenesis Research Laboratory, Department \\ of Cariology, Restorative Sciences and Endodontics, University of Michigan School of Dentistry; ${ }^{3}$ Department of Periodontics and \\ Oral Medicine; ${ }^{4}$ Department of Biomedical Engineering, University of Michigan College of Engineering; ${ }^{5}$ Department of \\ Otolaryngology, University of Michigan School of Medicine, Ann Arbor, MI, USA
}

BACKGROUND: Loco-regional spread of disease causes high morbidity and is associated with the poor prognosis of malignant oral tumors. Better understanding of mechanisms underlying the establishment of lymph node metastasis is necessary for the development of more effective therapies for patients with oral cancer. The aims of this work were to evaluate a possible correlation between endothelial cell $\mathrm{Bcl}-2$ and lymph node metastasis in patients with oral squamous cell carcinoma (OSCC), and to study signaling pathways that regulate $\mathrm{Bcl}-2$ expression in lymphatic endothelial cells.

METHODS: Endothelial cells were selectively retrieved from paraffin-embedded tissue sections of primary human OSCC from patients with or without lymph node metastasis by laser capture microdissection. RT-PCR was used to evaluate $\mathrm{BCl}-2$ expression in tumor-associated endothelial cells and in tumor cells. In vitro, mechanistic studies were performed to examine the effect of vascular endothelial growth factor (VEGF)-C on the expression of Bcl-2 in primary human lymphatic endothelial cells.

RESULTS: We observed that $\mathrm{Bcl}-2$ expression is upregulated in the endothelial cells of human oral tumors with lymph node metastasis as compared to endothelial cells from stage-matched tumors without metastasis. VEGF-C induced $\mathrm{Bcl}-2$ expression in lymphatic endothelial cells via VEGFR-3 and PI3k/Akt signaling. Notably, OSCC cells express VEGF-C and induce $\mathrm{Bcl}-2$ in lymphatic endothelial cells.

CONCLUSIONS: Collectively, this work unveiled a mechanism for the induction of $\mathrm{Bcl}-2$ in lymphatic endothelial cells and suggested that endothelial cell Bcl-2 contributes to lymph node metastasis in patients with oral squamous cell carcinoma.

J Oral Pathol Med (20I2) 4I: I24-130

Correspondence: Jacques E. Nör, DDS, MS, PhD, Dentistry, Otolaryngology, and Biomedical Engineering, University of Michigan, 1011 N. University Rm. 2309, Ann Arbor, MI 48109-1078, USA. Tel: 734936 9300, Fax: 734936 1597, E-mail: jenor@umich.edu Accepted for publication August 8, 2011
Keywords: angiogenesis; biomarkers; head and neck cancer; lymphangiogenesis; oral cancer; vascular endothelial growth factor

\section{Introduction}

The crosstalk between neovascular endothelial cells and tumor cells plays a major role in oral squamous cell carcinoma (OSCC) tumor growth and angiogenesis (1-3). We have reported that $\mathrm{Bcl}-2$ is a key regulator of this crosstalk $(1,2)$ and have reported a novel role for $\mathrm{Bcl}-2$ as a pro-angiogenic signaling molecule (4). However, the functional implications of the molecular interactions between lymphatic endothelial cells and OSCC cells are not clearly understood. Considering that OSCC frequently metastasize to the lymph nodes, a better understanding of the crosstalk between lymphatic endothelial cells and OSCC may lead to new therapies and to significant improvements in the management of patients with oral cancer.

It has been reported that vascular endothelial growth factor (VEGF)-C and its cognate receptor VEGFR-3 are expressed in head and neck squamous cell carcinomas (5). VEGF-C binds primarily to VEGFR-3, inducing lymphangiogenesis (6) and regulating several cellular functions involved in cancer progression $(5,7-9)$. Once activated, VEGFR-3 is phosphorylated and triggers signaling through the PI3k/Akt pathway $(10,11)$. The activation of the PI3k/Akt pathway can induce Bcl-2 expression in vascular endothelial cells (12). However, the role of the PI3k/Akt pathway in the regulation of VEGF-C-induced Bcl-2 expression in lymphatic endothelial cells remains unclear.

This study was designed to examine signaling pathways involved in the regulation of $\mathrm{Bcl}-2$ in lymphatic endothelial cells, and to evaluate the impact of endothelial cell $\mathrm{Bcl}-2$ expression in primary tumors on lymph node metastasis. Our results suggest that Bcl-2 expression levels in primary tumor-associated endothelial cells are correlated with the incidence of lymph node 
metastasis in patients with OSCC. Furthermore, this work revealed that VEGF-C induces Bcl-2 expression in lymphatic endothelial cells via VEGFR-3 signaling through the PI3k/Akt pathway.

\section{Materials and methods}

\section{Cell culture}

Primary human dermal lymphatic microvascular endothelial cells (HMVEC) and human dermal microvascular endothelial cells (HDMEC) were obtained from Lonza (Walkersville, MD, USA) and cultured in EGM$2 \mathrm{MV}$ (Lonza). The human oropharyngeal SCC cell lines (UM-SCC-11A, UM-SCC-11B, UM-SCC-17A, gift from Dr. T. Carey, University of Michigan) were cultured in Dulbecco's modified Eagle's medium (DMEM; Invitrogen, Carlsbad, CA, USA) supplemented with $10 \%$ fetal bovine serum and penicillin/streptomycin.

\section{Western blots}

VEGF-C and VEGFR-3 baseline expression levels were evaluated by Western blots using polyclonal anti-human VEGF-C and anti-human VEGFR-3 antibodies, respectively (Santa Cruz Biotechnology, Santa Cruz, CA, USA). Furthermore, HMVEC and HDMEC were serum-starved overnight, pretreated with 0-30 $\mu \mathrm{M}$ LY294002 (Sigma-Aldrich, St. Louis, MO, USA) for $1 \mathrm{~h}$, and then treated with $0-50 \mathrm{ng} / \mathrm{ml} \mathrm{rhVEGF-C} \mathrm{(R} \mathrm{\&}$ D Systems, Minneapolis, MN, USA) for 24 hours in the presence of LY2964002. Alternatively, HMVEC were exposed to serum-free conditioned media collected from the SCC cell lines for $24 \mathrm{~h}$. The role of VEGFR-3 in VEGF-C-induced Bcl-2 expression was evaluated with 0-10 $\mathrm{g} / \mathrm{ml}$ anti-human VEGFR-3 neutralizing antibody (Santa Cruz). Bcl-2 expression was evaluated by Western blot with the hamster anti-human Bcl-2 (BD Biosciences, San Jose, CA, USA), and phosphorylated and total Akt expression was also analyzed with respective polyclonal anti-human antibodies (Cell Signaling, Beverly, MA, USA).

\section{LCM and RT-PCR from human OSCC}

Six cases of OSCC with known clinical outcomes were studied here (Table 1). They included three metastatic $(\mathrm{M}+\mathrm{OSCC})$ and three non-metastatic (M- OSCC) tumors. The limited number of cases in each group is explained by the fact that the samples included in this study strictly met the following inclusion criteria: (A) they should be retrieved from patients who had surgical neck resection and lymph node immunohistochemical confirmation with analysis for cytokeratins (Fig. 1); and (B) the laser capture microdissection (LCM)-retrieval should generate at least 1500 endothelial cells and 10000 tumor cells located exclusively in the advancing tumor front. Immunohistochemical staining for Factor VIII (1:500; Neomarkers, Fremont, CA, USA) was performed to identify vascular endothelial cells in preparation for the LCM, as described (1). Endothelial cells and tumor cells were retrieved from each tissue section from the three independent tumors and pooled for RT-PCR analysis according to each experimental condition. Total RNA was isolated using Trizol reagent (Invitrogen) and purified with RNAeasy Mini Kits (Qiagen Inc., Valencia, CA, USA). SuperScript one-step reverse-transcription-PCR (RT-PCR) was performed using the Platinum Taq kit (Invitrogen). The following are the primer sequences: E-cadherin (sense 5'-TGGAC AGGGAGGATTTTGAG-3'; antisense 5'-CTTGAGC CCCAGAGTTTGAG-3'; 385 bp amplicon); Bcl-2 (sense 5'-CTGCGAAGAACCTTGTGTGA-3'; antisense 5'-TGTCCCTACCAACCAGAAGG-3'; 214 bp amplicon); and GAPDH sense 5'-CATGGCCTCCAA GGAGTAAG-3'; antisense 5'-AGGGGTCTACAGGC AACTG-3'; 683 bp).

\section{Results}

Bcl-2 expression in the endothelial cells of metastatic tumors

A stepwise method was used to selectively retrieve endothelial cells or OSCC cells from paraffin-embedded tissue sections (Fig. 2). The first step aims at the elimination of luminal contents (Fig. 2B). This is followed by retrieval of tumor-associated endothelial cells (Fig. 2C), and retrieval of tumor cells (Fig. 2D). This method results in a relatively pure RNA preparation, as demonstrated by the analysis of E-cadherin expression, a well-known marker for cells with epithelial origin (Fig. 2E,F; Fig. S1). Indeed, while the tumor cell RNA preparations showed strong positive expression of E-cadherin, the endothelial cell RNA preparations did not. Once the purity of the RNA preparations was confirmed, we evaluated Bcl-2 expression levels in the endothelial cells of tumors from patients with or without lymph node metastasis. We observed that Bcl-2 was

Table 1 Personal data, staging, histological grade, and number of metastatic lymph nodes in the oral squamous cell carcinoma cases evaluated here. These tumors were located in the tongue and/or floor of the mouth, as determined by clinical and histological evaluation

\begin{tabular}{lllll}
\hline Patient & \multicolumn{1}{c}{ Personal data } & TNM & Histological grade & LN involvement (number of lymph nodes) \\
\hline $\mathrm{M}(+) 1$ & Male, White, 58 years old & T2N2M0 & I & L2 (2), L3 (2), L4 (2) \\
$\mathrm{M}(+) 2$ & Male, White, 61 years old & T2N1M0 & III & L2 (4) \\
$\mathrm{M}(+) 3$ & Male, White, 69 years old & T4N2aM0 & III & RL1 (3), RL2 (3), RL3 (1); LL1 (2), LL2 (4) \\
$\mathrm{M}(-) 1$ & Female, White, 63 years old & T2N0M0 & II & None \\
$\mathrm{M}(-) 2$ & Male, White, 48 years old & T4aN0M0 & III & None \\
$\mathrm{M}(-) 3$ & Male, White, 62 years old & T2N0M0 & III & None \\
\hline
\end{tabular}

RL, right side and level; LL, left side and level; L, level. 

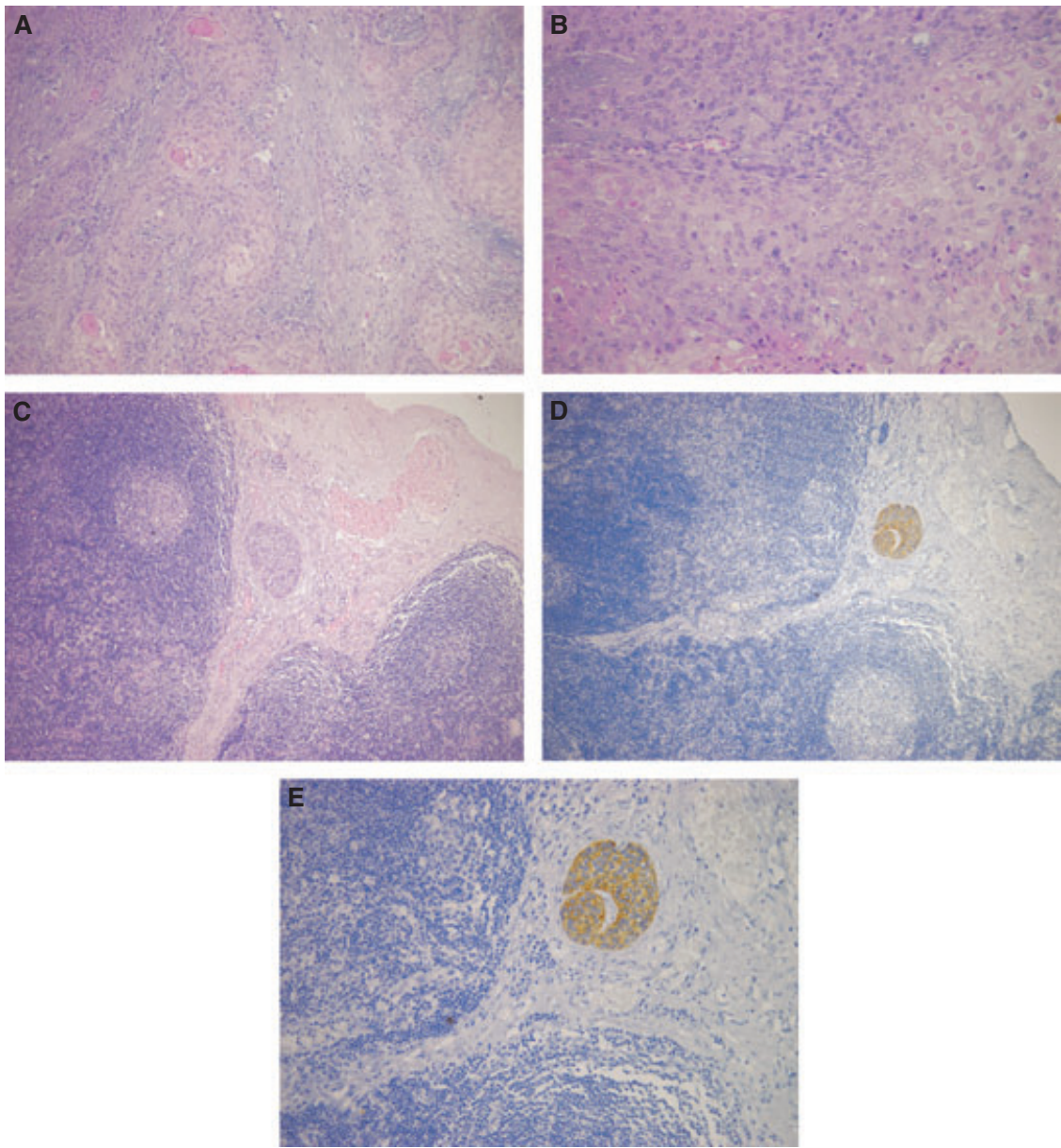

Figure 1 Histopathological and immunohistochemical features of an oral squamous cell carcinoma with lymph node metastasis. (A-B) Representative tumor front of a primary M+ OSSC case evaluated here (A - HE, 100×; B - HE, 200×). (C-D). Lymph node metastasis of OSSC showing a nest of malignant keratinocytes (C - HE, 100×) positive for AE1/AE3 antibody (D - AE1/AE3, 200×).
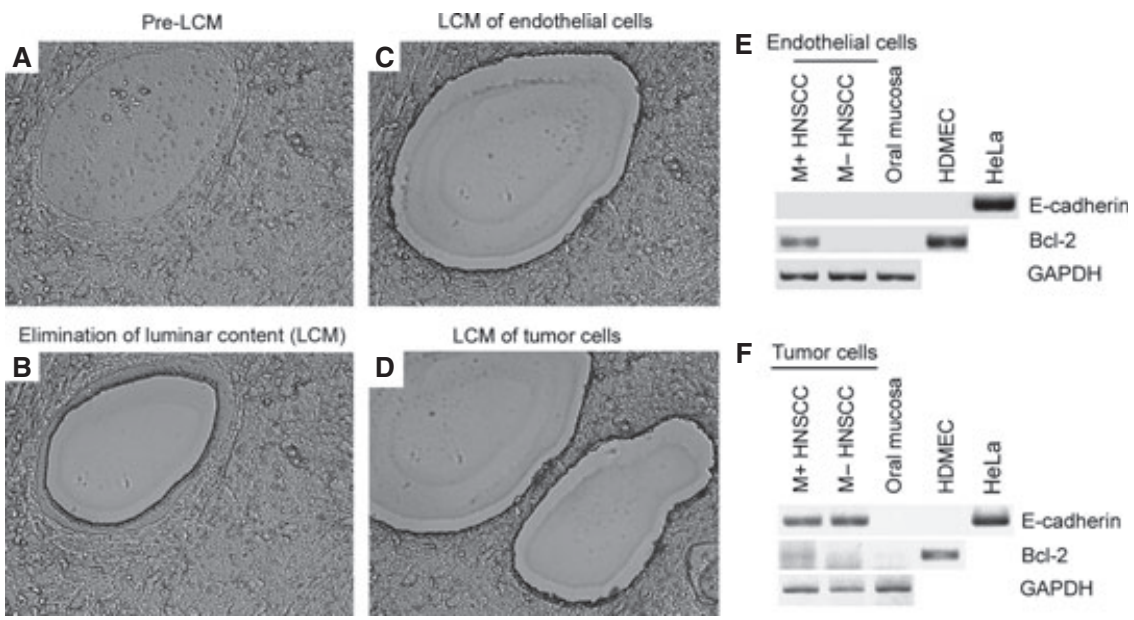

Figure 2 Bcl-2 is expressed in tumor-associated endothelial cells of metastatic human oral squamous cell carcinomas (OSCC). (A-D) Step-by-step characterization of the technique used for retrieval of either tumor cells or endothelial cells from paraffin-embedded tissue sections by laser capture microdissection (LCM). (E) RT-PCR analysis of Bcl-2 and E-cadherin expression in the endothelial cells of metastatic $(\mathrm{M}+)$ or non-metastatic (M-) OSCC. (F) RT-PCR analysis of Bcl-2 and E-cadherin expression in the tumor cells of metastatic (M +) or non-metastatic (M-) OSCC. (E,F) Controls were vascular endothelial cells captured by LCM from normal oral mucosa (EC, oral mucosa), human dermal microvascular endothelial cells (HDMEC), and human cervical cancer cell line (HeLa). 
strongly expressed in tumor-associated endothelial cells of patients with metastasis, but not in patients without metastasis (Fig. 2E). Interestingly, Bcl-2 expression was undetectable in the OSCC cells (Fig. 2F).

\section{VEGF-C signals through VEGFR-3 to induce Bcl-2 in lymphatic endothelial cells}

To understand potential mechanisms regulating $\mathrm{Bcl}-2$ in lymphatic endothelial cells, we studied the activity of the VEGF-C and VEGFR-3 signaling pathways. While lymphatic endothelial cells strongly express VEGFR-3, vascular endothelial cells and three OSCC cell lines did not express this receptor (Fig. 3A). In contrast, all cells examined here expressed VEGF-C (Fig. 3A). Bcl-2 expression was potently induced by rhVEGF-C in lymphatic endothelial cells (Fig. 3B). The growth factor milieu secreted by OSCC potently induced Bcl-2 expression in lymphatic endothelial cells (Fig. 3C). Notably, VEGF-C-induced Bcl-2 expression is mediated by VEGFR-3 signaling in lymphatic endothelial cells (Fig. 3D).

\section{Akt mediates VEGF-C-induced Bcl-2 expression in lymphatic endothelial cells}

To evaluate the role of Akt on the regulation of $\mathrm{Bcl}-2$ expression in lymphatic endothelial cells, we used LY294002, an inhibitor of the PI3k-Akt signaling. VEGF-C induced rapid and potent phosphorylation of Akt in lymphatic endothelial cells (Fig. 4A). As expected, LY294002 blocked VEGF-C-induced Akt phosphorylation in these cells (Fig. 4A). Blockade of Akt phosphorylation abrogated VEGF-C-induced Bcl-2 expression in lymphatic endothelial cells, demonstrating the requirement of Akt signaling in this process (Fig. 4B). In contrast, VEGF-C had no effect on Akt phosphorylation and Bcl-2 expression in dermal endo-

\section{Discussion}

It is well known that the control of loco-regional disease constitutes a major challenge in the management of patients with oral cancer. A complicating factor is that very little is known about the mechanisms underlying the displacement of OSCC from the primary tumor site and the beginning of the cellular processes that result in lymph node metastasis. Lymphatic vessels can be found within certain cancer types $(13,14)$. Notably, emerging evidence has shown that cancer cells invade into local lymph nodes mainly via peritumoral lymphatic vessels (15). It has been recently shown that the crosstalk between cancer cells and lymphatic endothelium induces changes in the lymphatics that may contribute to metastatic spread (16). Indeed, lymphatic endothelial cells activated by highly metastatic cancer cells displayed aberrant behaviors and secrete chemokines that facilitate cancer cell migration (17). Here, we observed that $\mathrm{Bcl}-2$ is highly expressed in tumor-associated endothelial cells of oral cancer patients with lymph node metastasis, while it is undetectable in the endothelial cells of patients that did not metastasize. These findings enhance the understanding of mechanisms of OSCC metastasis and may have implications to the clinical management of patients with oral cancer.

We have previously shown that endothelial cell Bcl-2 signaling promotes head and neck tumor growth (1) and induces tumor cell motility via the CXC chemokine pathway (18). These findings provided the rationale for the current exploration of the role of endothelial cell Bcl-2 on the metastatic spread of head and neck
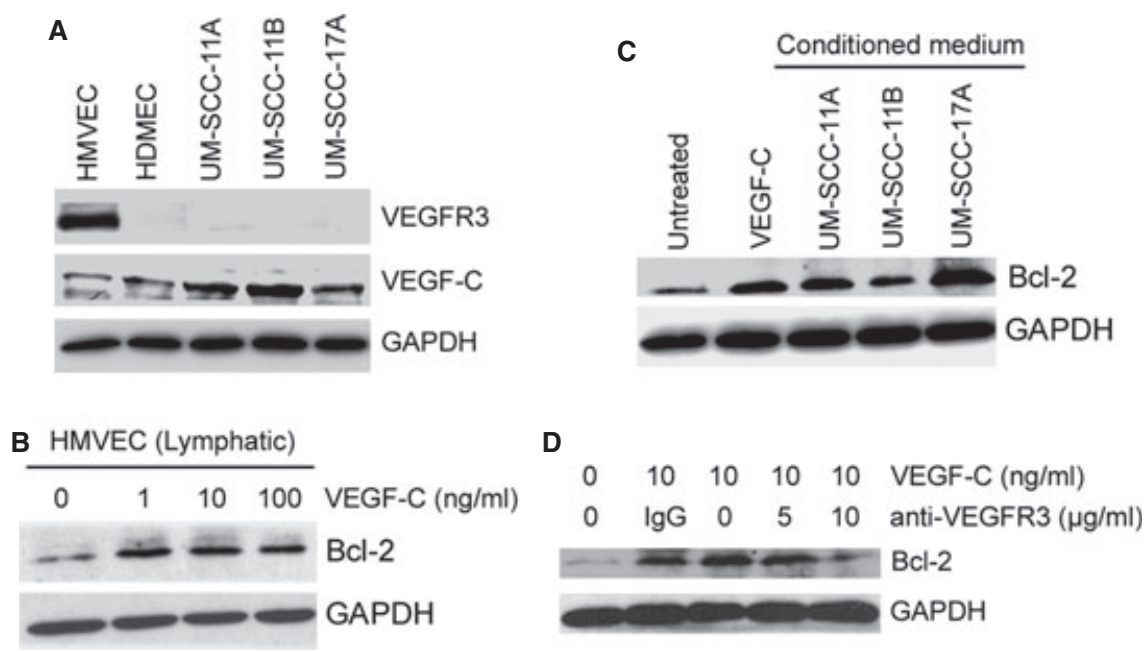

Figure 3 Vascular endothelial growth factor (VEGF)-C and the growth factor milieu secreted by oral squamous cell carcinoma (OSCC) induce Bcl-2 expression in lymphatic endothelial cells. (A) Western blot for the evaluation of baseline VEGF-C and VEGFR-3 expression in human lymphatic endothelial cells (HMVEC), human dermal microvascular endothelial cells (HDMEC), and in three OSCC cell lines (UM-SCC-11A, UM-SCC-11B, UM-SCC-17A); (B) Western blot to study the effect of rhVEGF-C on Bcl-2 expression in lymphatic endothelial cells. (C) Western blot for evaluation of the effect of 24-h conditioned medium from three OSCC cells lines on Bcl-2 expression in lymphatic endothelial cells. (D) Western blot to determine the role of VEGFR-3 on VEGF-C-induced Bcl-2 in lymphatic endothelial cells. Cells were exposed to $10 \mathrm{ng} / \mathrm{ml}$ rhVEGF-C in the presence of increasing concentrations of neutralizing anti-VEGFR3 antibody. 


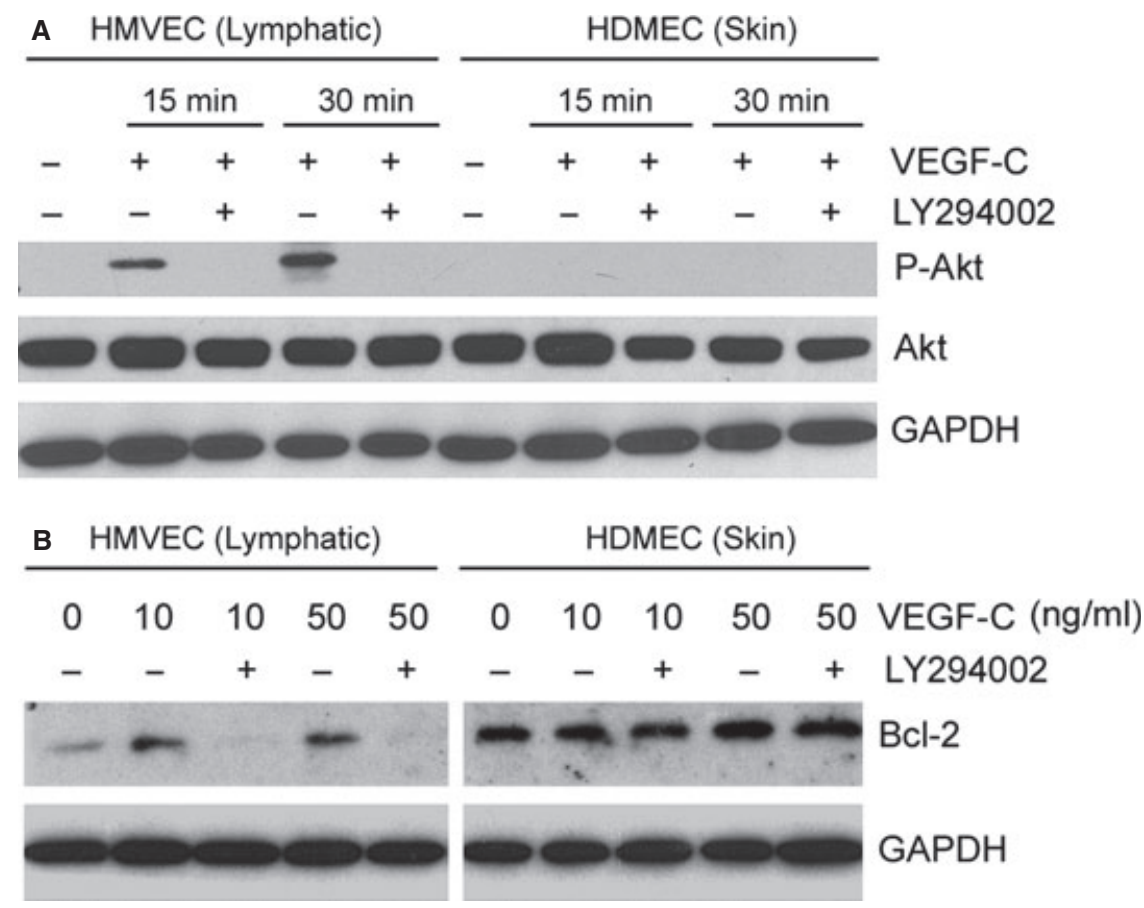

Figure 4 Vascular endothelial growth factor (VEGF)-C induces Bcl-2 expression via PI3k/Akt signaling in lymphatic endothelial cells. (A,B) Lymphatic and dermal (vascular) endothelial cells were exposed to VEGF-C for 15 or $30 \mathrm{~min}$ in the presence (or absence) of the inhibitor of PI3k/Akt signaling, LY294002. (A) Western blots for the evaluation of the expression of total and phosphorylated Akt. (B) Western blot for the evaluation of Bcl-2 expression.

squamous cell carcinomas (HNSCC). Since we found that endothelial cell Bcl-2 expression levels were correlated with lymph node metastasis, and assuming that lymph node metastases would be primarily driven by invasion through lymphatic vessels, we focused the mechanistic studies included here on the analyses of lymphatic endothelial cells, using vascular endothelial cells as controls. We observed that all OSCC cell lines evaluated here express VEGF-C. VEGF-C expression has been correlated with lymphatic metastatic potential and invasiveness of cancer cells (18-21). Zhuang et al. have shown that VEGF-C is expressed by highly metastatic OSCC cells $(16,17)$. We observed that VEGF-C, as well as the growth factor milieu secreted by several OSCC cell lines, induced Bcl-2 expression in lymphatic endothelial cells. These data are in line with the observation that VEGF-C induces the survival of leukemic tumor cells (7). Notably, the VEGF-C/VEGFR3 axis activation has been associated with lymphangiogenesis (19), angiogenesis (22), and endothelial cell survival (23). Collectively, these observations suggest that VEGF-C augments the lymphatic component of oral tumors by enhancing the Bcl-2 expression in the lymphatic endothelial cells.

It has been shown that VEGF-C activates the PI3k/Akt pathway through VEGFR3 (7, 10). Here, we showed that VEGF-C induces Akt phosphorylation and Bcl-2 expression in VEGFR3-expressing lymphatic endothelial cells, but not in dermal endothelial cells that do not express VEGFR3. These data suggest that the effects of VEGF-C on Bcl-2 are specific to the lymphatic system. Further, we observed that signaling through
VEGFR3 and the phosphorylation of Akt are required for $\mathrm{Bcl}-2$ expression in lymphatic endothelial cells. Collectively, these findings unveil a pathway for the regulation of lymphatic endothelial cell survival that is orchestrated by Bcl-2.

The gene expression analysis performed with cells retrieved from human OSCC provides clinical relevance to our laboratory results. Tumor-associated endothelial cells from metastatic OSCC cases expressed Bcl-2, while the endothelial cells from the tumors of patients that did not exhibit metastasis did not express Bcl-2. To the best of our knowledge, this is the first demonstration of a correlation between primary tumor-associated endothelial cell Bcl-2 expression and the presence of lymph node metastasis. We speculate that $\mathrm{Bcl}-2$ may contribute to the metastatic spread to lymph nodes by the following: (i) Increasing the survival of tumor-associated endothelial cells in OSCC; (ii) Triggering signaling pathways that result in enhanced secretion of chemotactic factors by endothelial cells; and (iii) Enhancing the invasive phenotype of OSCC cells. Notably, we have demonstrated that Bcl-2 induces expression of CXCL1 and CXCL8 in vascular endothelial cells (4) and that these factors signal through CXCR2 to enhance the invasiveness of head and neck squamous cell carcinoma cells (18). Our laboratory is currently performing studies to better understand the functional role of Bcl-2 in tumorassociated lymphatic endothelial cells.

Taken together, our results unveiled a signaling pathway that may have significant implications to the regulation of lymphangiogenesis and metastatic spread of oral cancers (Fig. 5). These studies suggest that Bcl-2 


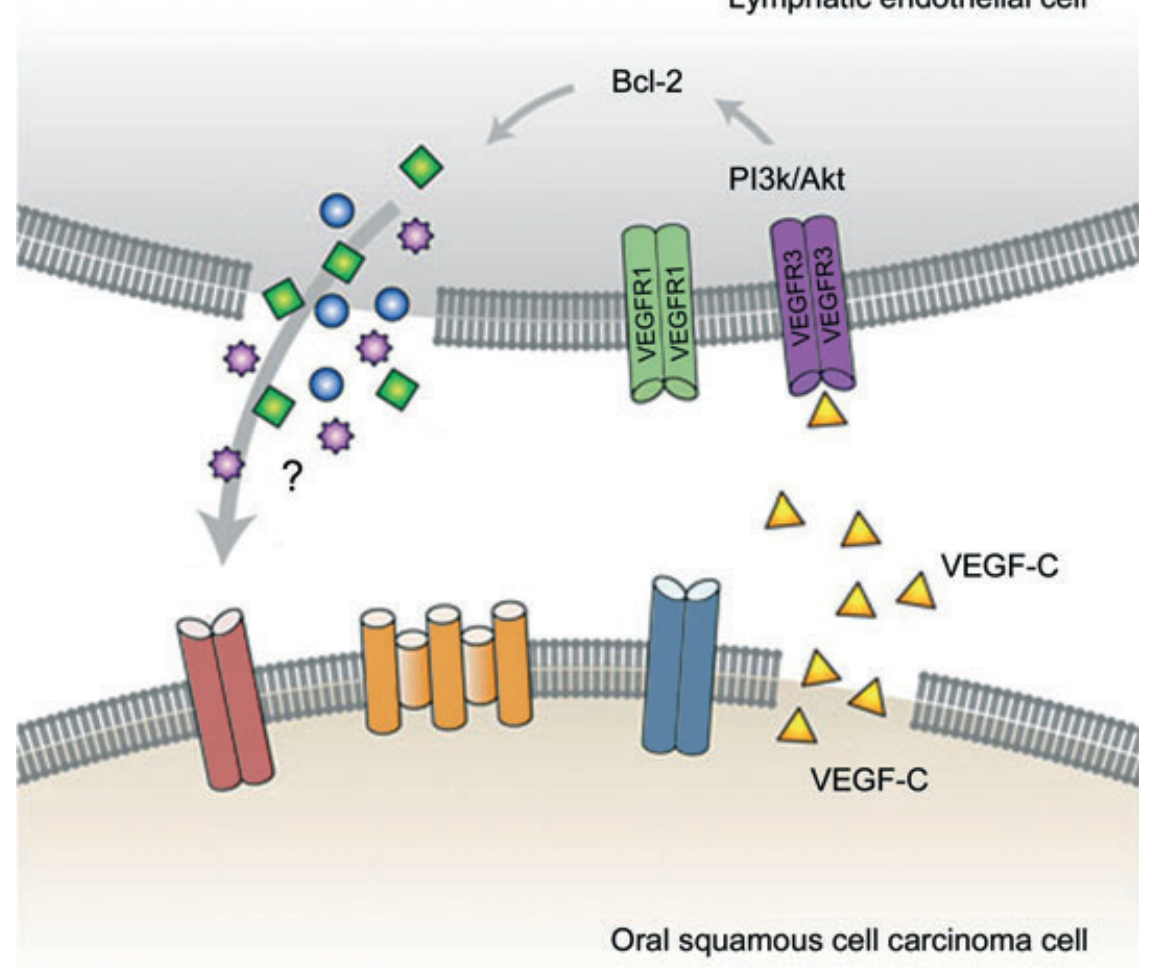

Figure 5 Diagram proposing a model for the crosstalk between oral squamous cell carcinoma (OSCC) cells and lymphatic endothelial cells. OSCC cells secrete vascular endothelial growth factor (VEGF)-C that signals through VEGFR-3 to induce phosphorylation of Akt in lymphatic endothelial cells. Activation of PI3k/Akt signaling induces Bcl-2 expression in lymphatic endothelial cells. We hypothesize that Bcl-2 expression results in the upregulation of downstream target proteins that are secreted by lymphatic endothelial cells and that may affect tumor cell invasive behavior. However, the latter is speculation based on pilot studies from our laboratory and therefore is labeled with a question mark.

expression in the endothelial cells of primary tumors might serve as a biomarker for predictions about the metastatic potential of OSCC. These data also suggest potential targets for the treatment of OSCCs. Particularly, targeted therapeutic inhibition of Bcl-2 may have the following complementary, and perhaps synergistic, effects: (i) Inhibition of lymphangiogenesis in OSCC; (ii) Inhibition of vascular angiogenesis; and (iii) Direct inhibition of tumor cell survival. The recent discovery of highly potent and specific small molecule inhibitors of Bcl-2 (24) raises the exciting possibility of using these drugs in the management of patients with oral cancer.

\section{Supporting Information}

Additional Supporting Information may be found in the online version of this article:

Figure S1 Purity and specificity of the RNA retrieved from paraffin-embedded tissue sections. RT-PCR analysis of the expression of E-cadherin (marker of cells of epithelial origin) and VEGFR2 (endothelial cell marker) in the RNA prepared from the endothelial cell fraction and from the tumor cell fraction. Controls were human dermal microvascular endothelial cells (HDMEC), from the University of Michigan Head and Neck Squamous Cell Carcinoma cell line (UM-SCC-11A), and a human cervical cancer cell line (HeLa).
Please note: Wiley-Blackwell are not responsible for the content or functionality of any supporting materials supplied by the authors. Any queries (other than missing material) should be directed to the corresponding author for the article.

\section{References}

1. Kaneko T, Zhang Z, Mantellini MG, et al. Bcl-2 orchestrates a cross-talk between endothelial and tumor cells that promotes tumor growth. Cancer Res 2007; 67: 9685-93.

2. Neiva KG, Zhang Z, Miyazawa M, et al. Cross talk initiated by endothelial cells enhances migration and inhibits anoikis of squamous cell carcinoma cells through STAT3/Akt/ERK signaling. Neoplasia 2009; 11: 583-93.

3. Nör JE, Christensen J, Liu J, et al. Up-regulation of Bcl-2 in microvascular endothelial cells enhances intratumoral angiogenesis and accelerates tumor growth. Cancer Res 2001; 61: 2183-8.

4. Karl E, Warner K, Zeitlin B, et al. Bcl-2 acts in a proangiogenic signaling pathway through nuclear factorkappaB and CXC chemokines. Cancer Res 2005; 65: 50639.

5. Neuchrist C, Erovic BM, Handisurya A, et al. Vascular endothelial growth factor $\mathrm{C}$ and vascular endothelial growth factor receptor 3 expression in squamous cell carcinomas of the head and neck. Head Neck 2003; 25: 464-74.

6. Kishimoto K, Sasaki A, Yoshihama Y, et al. Expression of vascular endothelial growth factor-C predicts regional 
lymph node metastasis in early oral squamous cell carcinoma. Oral Oncol 2003; 39: 391-6.

7. Dias S, Choy M, Alitalo K, et al. Vascular endothelial growth factor (VEGF)-C signaling through FLT-4 (VEGFR-3) mediates leukemic cell proliferation, survival, and resistance to chemotherapy. Blood 2002; 99: 2179-84.

8. Saintigny $\mathrm{P}$, Kambouchner M, Ly M, et al. Vascular endothelial growth factor-C and its receptor VEGFR-3 in non-small-cell lung cancer: concurrent expression in cancer cells from primary tumour and metastatic lymph node. Lung Cancer 2007; 58: 205-13.

9. $\mathrm{Su}$ JL, Yen CJ, Chen PS, et al. The role of the VEGFC/VEGFR-3 axis in cancer progression. Br J Cancer 2007; 96: $541-5$.

10. Makinen T, Veikkola T, Mustjoki S, et al. Isolated lymphatic endothelial cells transduce growth, survival and migratory signals via the VEGF-C/D receptor VEGFR-3. EMBO J 2001; 20: 4762-73.

11. Wissmann C, Detmar M. Pathways targeting tumor lymphangiogenesis. Clin Cancer Res 2006; 12: 6865-8.

12. Cai J, Ahmad S, Jiang WG, et al. Activation of vascular endothelial growth factor receptor-1 sustains angiogenesis and Bcl-2 expression via the phosphatidylinositol 3kinase pathway in endothelial cells. Diabetes 2003; 52: 2959-68.

13. Kowalski LP, Sanabria A. Elective neck dissection in oral carcinoma: a critical review of the evidence. Acta Otorhinolaryngol Ital 2007; 27: 113-7.

14. Okada Y. Relationships of cervical lymph node metastasis to histopathological malignancy grade, tumor angiogenesis, and lymphatic invasion in tongue cancer. Odontology 2010; 98: 153-9.

15. Zhang Z, Helman JI, Li LJ. Lymphangiogenesis, lymphatic endothelial cells and lymphatic metastasis in head and neck cancer - a review of mechanisms. Int J Oral Sci 2010; 2: $5-14$

16. Zhuang Z, Jian P, Longjiang L, et al. Altered phenotype of lymphatic endothelial cells induced by highly metastatic OTSCC cells contributed to the lymphatic metastasis of OTSCC cells. Cancer Sci 2010; 101: 686-92.

17. Zhuang Z, Jian P, Longjiang L, et al. Oral cancer cells with different potential of lymphatic metastasis displayed distinct biologic behaviors and gene expression profiles. J Oral Pathol Med 2010; 39: 168-75.

18. Warner KA, Miyazawa M, Cordeiro MM, et al. Endothelial cells enhance tumor cell invasion through a crosstalk mediated by CXC chemokine signaling. Neoplasia 2008; 10: 131-9.

19. Alitalo K, Carmeliet P. Molecular mechanisms of lymphangiogenesis in health and disease. Cancer Cell 2002; 1: 219-27.

20. Nakazato T, Shingaki S, Kitamura N, et al. Expression level of vascular endothelial growth factor-C and -A in cultured human oral squamous cell carcinoma correlates respectively with lymphatic metastasis and angiogenesis when transplanted into nude mouse oral cavity. Oncol Rep 2006; 15: 825-30.

21. Siriwardena BS, Kudo Y, Ogawa I, et al. VEGF-C is associated with lymphatic status and invasion in oral cancer. J Clin Pathol 2008; 61: 103-8.

22. Ding MX, Lin XQ, Fu XY, et al. Expression of vascular endothelial growth factor-C and angiogenesis in esophageal squamous cell carcinoma. World J Gastroenterol 2006; 12: $4582-5$.

23. Zhao B, Smith G, Cai J, et al. Vascular endothelial growth factor C promotes survival of retinal vascular endothelial cells via vascular endothelial growth factor receptor-2. $\mathrm{Br}$ J Ophthalmol 2007; 91: 538-45.

24. Zeitlin BD, Zeitlin IJ, Nör JE. Expanding circle of inhibition: small-molecule inhibitors of Bcl-2 as anticancer cell and antiangiogenic agents. J Clin Oncol 2008; 26: 4180-8.

\section{Acknowledgements}

This work was supported by a grant from CAPES (Brazilian Government) to S. Tarquinio; by grant P50-CA97248 (University of Michigan Head and Neck SPORE) from the NIH/NCI, and grants R01-DE15948, R01DE16586, R21-DE19279 from the NIH/NIDCR to J. Nör.

\section{Conflict of interest statement}

The authors have no conflict of interest. 